\title{
Design, Synthesis, Hypoglycemic Activity and Molecular Docking Studies of 3-substituted-5- [(furan-2-yl)-methylene]-thiazolidine-2,4-dione Derivatives
}

\author{
Karumanchi Srikanth Kumar', Atmakuri Lakshmana Rao, ${ }^{1, *}$, Dachuru Rama Sekhara Reddy² \\ 1'Department of Pharmaceutical Chemistry, VV Institute of Pharmaceutical Sciences, Gudlavalleru, Andhra Pradesh, INDIA. \\ ${ }^{2}$ Department of Chemistry, Krishna University, Machilipatnam, Andhra Pradesh, INDIA.
}

\begin{abstract}
Background: From the wide range of previous literature studies indicated that thiazolidinedione's reacts with substituted benzaldehydes undergoes knoevenagel condensation gives respective arylidene derivatives. In our attempt all the titled compounds were designed and developed by replacement of substituted benzaldehydes with furan- 2-aldehyde, so that furan moiety was introduced in the molecule. Materials and Methods: 5-[(furan-2-yl)-methylene]-thiazolidine-2,4-dione was prepared via knoevenagel condensation by the reaction of thiazolidine-2,4-dione and furfural. Further it was coupled with various alkyl/ aryl halides in alcoholic potassium hydroxide to produce various derivatives $2 \mathrm{a}-2 \mathrm{j}$. The titled compounds furthermore prepared by microwave assisted synthesis technique. Synthesized compounds were analysed by physical and spectral characterization methods. Developed furan bearing thiazolidine-2,4-diones were evaluated for in-vivo hypoglycemic property. Molecular docking analysis was carried out to observe the binding interaction of designed ligands at PPAR $\gamma$ target receptor protein. Results and Conclusion: Microwave irradiation technique produced high yield at less reaction time in comparison with traditional conventional method. In-vivo hypoglycemic activity evaluation revealed that, electron releasing groups $(-\mathrm{OH}$ and $-\mathrm{OCH} 3)$ containing compounds $2 \mathrm{~d}$ and $2 \mathrm{~g}$ found to possess significant activity in acute study as well as in chronic study. Even the molecular docking studies at PPAR $\gamma$ receptor protein (PDB ID$2 P R G$ ), electron releasing groups containing compounds $2 \mathrm{~d}$ and $2 \mathrm{~g}$ exhibit significant binding affinity having high binding energy of $-9.02 \mathrm{kcal} / \mathrm{mol}$ and $-8.61 \mathrm{kcal} / \mathrm{mol}$ when compared with standard ligand rosiglitazone.
\end{abstract}

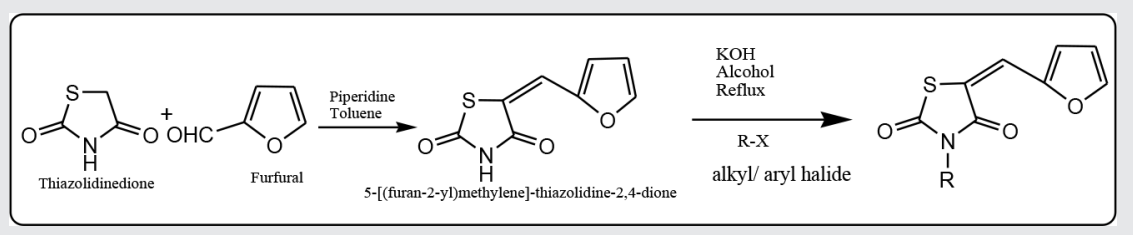

Key words: Thiazolidinedione derivatives, Synthesis, Hypoglycemic Activity, Molecular Docking, PDB ID-2PRG.

\section{INTRODUCTION}

Diabetes mellitus (DM) is universally widespread chronic metabolic disorder during which elevated blood sugar levels take place over a prolonged period of time and symptoms comprises recurrent urination, increased hunger and thirsty. DM is allied with rigorous degenerative complications for instance nephropathy, cataract, neuropathy, accelerated atherosclerosis, retinopathy and stroke and increased the risk of myocardial infarction. Onset of these pathologies is a remarkable event throughout both
Submission Date: 01-04-2020; Revision Date: 09-07-2020; Accepted Date: 28-12-2020

DOI: 10.5530/ijper.55.1.30 Correspondence:

Dr. A. Lakshmana Rao Professor, Department of Pharmaceutical Chemistry, VV Institute of Pharmaceutical Sciences,

Gudlavalleru-521356, Andhra Pradesh, INDIA. Phone no: +919848779133 Email id: dralrao@gmail.com

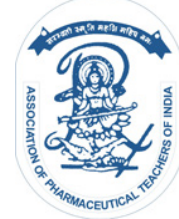

www.ijper.org 
type 1 and type 2 diabetes. Still severe challenging therapeutically problems for prevention and control for diabetic patients as they stand for the leading causes of morbidity and mortality. ${ }^{1}$ Long ago type 2 diabetes seen only in adults, but presently the incident often even in children. In general, up to $80 \%$ of insulin stimulated glucose disposal takes place in the skeletal muscles, which is considered the foremost site of insulin resistance in type 2 diabetes. ${ }^{2}$ Worldwide the incidence of diabetes in all age groups was expected to be $4.4 \%$ by the year 2030 . By the year 2030, it may rise up to 366 million. The rate of diabetes frequency is high in men than in women. By the year 2030, urban diabetes population is expected to double in the developing countries than in 2000. Across the worldwide the majority demographic transform to diabetes frequency appears to exist an increase in the diabetic people proportion greater than 65 years of age. Thus, there is a demand in developing novel drug candidates in this region. ${ }^{3}$

Thiazolidine-2,4-dione (TZD) is the most important heterocyclic moiety with much attention particularly in the treatment of type 2 diabetes. It is a 5 -membered unsaturated heterocyclic compound consists of $\mathrm{S}$ and $\mathrm{N}$ heteroatoms at the $1^{\text {st }}$ and $3^{\text {rd }}$ position of the ring respectively and has two ketone functional groups at $2^{\text {nd }}$ and $4^{\text {th }}$ positions respectively. Troglitazone, ciglitazone, pioglitazone, rosiglitazone are the TZD class of drugs possessing insulin sensitizing property. In the year 2000, troglitazone was withdrawn from the market owing to hepatotoxicity. TZDs produce hypoglycemic activity significantly by lowering the blood glucose levels through the activation of peroxisomal proliferator activated receptors gamma type (PPAR $\gamma) .^{4}$ TZDs improve the insulin action and promotes utilization of glucose in peripheral tissues. The accurate mechanism of TZDs still not been elucidated, but expected to show agonistic properties by binding with $\operatorname{PPAR} \gamma$ nuclear receptors. ${ }^{5}$ Thiazolidinedione's also known as glitazones, act by binding to PPAR $\gamma$ receptors, type of nuclear receptors regulates glucose and fat metabolism. Those molecules correct hyperglycemia and hyperinsulinemia in several animal models of NIDDM. Glucose clamp studies have clearly shown an improvement of insulin-induced glucose utilization (in skeletal muscle). TZDs exert their antidiabetic effects through activation of gamma isoform of the peroxisomal proliferator-activated receptor $(\operatorname{PPAR} \gamma)$ leads to alter the transcription of several genes involved in glucose and lipid metabolism and energy balance. TZDs reduce insulin resistance in adipose tissue, muscle and in the liver. However, PPAR $\gamma$ is predominantly expressed in adipose tissue. It is possible that the effect of TZDs on insulin resistance in muscle and liver is promoted via endocrine signaling from adipocytes. Although there are still many unknown factors about the mechanism of action of TZDs in type 2 diabetes, it is clear that these agents have the potential to benefit the "insulin resistance syndrome" associated with the disease. Hence, TZDs may have potential benefits on the secondary complications of type 2 diabetes. ${ }^{6,7}$

Recent developments in the area of TZDs found to possess an extensive array of biological actions for the instance antidiabetic, ${ }^{8,9}$ protein tyrosine phosphatise $1 \mathrm{~B}$ inhibitory, ${ }^{10,11}$ 15-hydroxyprostaglandin dehydrogenase inhibitory, ${ }^{12}$ hypolipidemic, ${ }^{13,14}$ aldose reductase inhibitory, ${ }^{15,16}$ anti-inflammatory, ${ }^{17,18}$ antimicrobial, ${ }^{19,20}$ antitubercular,,$^{21,22}$ antioxidant, ${ }^{23,24}$ and antitumor activities. ${ }^{25}$ From the wide range of previous literature studies indicated that thiazolidinedione's reacts with substituted benzaldehydes undergoes knoevenagel condensation gives respective arylidene derivatives. In our attempt all the titled compounds were designed and developed by replacement of substituted benzaldehydes with furan-2-aldehyde, so that furan moiety was introduced in the molecule.

\section{MATERIALS AND METHODS}

Commercial Merck grade reagents and solvents were procured and further used without purification. E. Merck grade silica gel 60GF-254 precoated thin layer chromatography (TLC) plates procured and used to monitor the reaction progress and completion. TLC spots were observed using ultraviolet light of UV-cabinet and in iodine chamber. Fourier Transform Infrared Spectrometer (FT-IR) spectra were recorded with a Bruker FT-IR analyzer spectrophotometer by compression of compound with anhydrous $\mathrm{KBr}$ under vacuum using the $\mathrm{KBr}$ pressed pellet technique. Chemical shifts in $\delta$, ppm of proton nuclear magnetic resonance ( $\left.{ }^{1} \mathrm{H}-\mathrm{NMR}\right)$ and carbon-13 nuclear magnetic resonance $\left({ }^{13} \mathrm{C}-\mathrm{NMR}\right)$ spectra were recorded on a Bruker AMX-400 MHz spectrometer using tetramethylsilane as reference standard and deuterated dimethyl sulfoxide (DMSO) as solvent. Mass spectra were recorded in Agilent LC-MSD-1200 mass spectrometer. Round bottom flask connected with reflux condenser setup was used for conventional synthesis on REMI magnetic stirrer with thermostat. Raga's scientific microwave system (RGSSIRR model) having different power levels (140W to $700 \mathrm{~W}$ ) was used for microwave assisted synthesis. Electrical melting point apparatus was used to determine melting point and were uncorrected. In the in-vivo hypoglycemic study wistar albino rats were used 
by dividing them into different groups and each group contained 6 rats $(n=6)$.

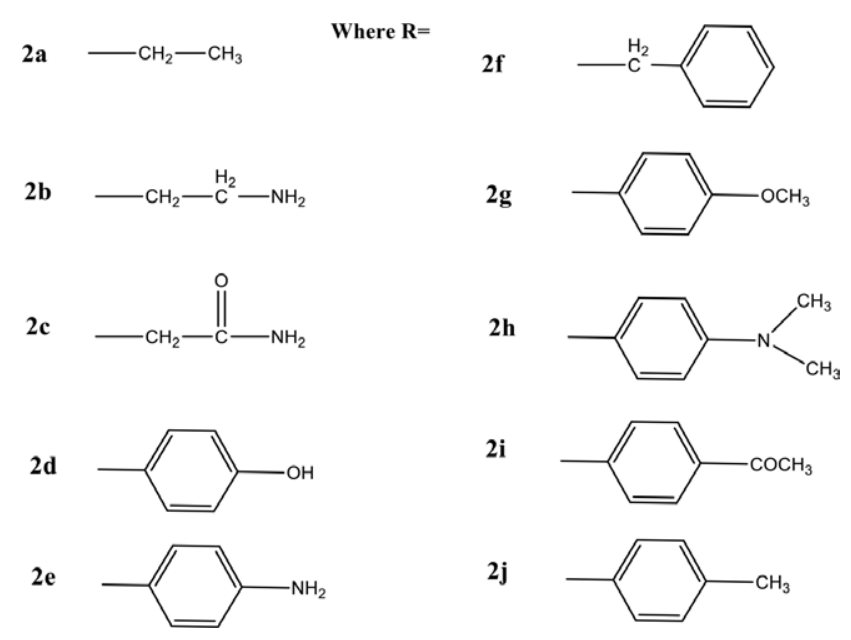

\section{Experimental}

Synthesis of 5-[(furan-2-yl)-methylene]-thiazolidine-2,4dione (1)

\section{Conventional synthesis}

Mixture of equal molar concentrations $(0.01 \mathrm{~mol})$ of thiazolidine-2,4-dione and furfural was taken in $8 \mathrm{ml}$ of toluene. To the reaction mixture, $0.4 \mathrm{ml}$ of piperidine was added, refluxed at $110-120^{\circ} \mathrm{C}$ for about $8 \mathrm{hr}$. Chromatographic TLC method was used to observe the reaction progress and completion. At room temperature the reaction mixture was cooled, add $1 \mathrm{M} \mathrm{HCl}$ solution and ice-water. Resulted solid precipitate content was filtered, washed from cold water followed by dry toluene. Crude product was recrystallized from ethanol. ${ }^{26}$

\section{Microwave synthesis}

$0.4 \mathrm{ml}$ of piperidine was added to $0.01 \mathrm{~mol}$ equimolar concentrations of thiazolidine-2,4-dione and furfural

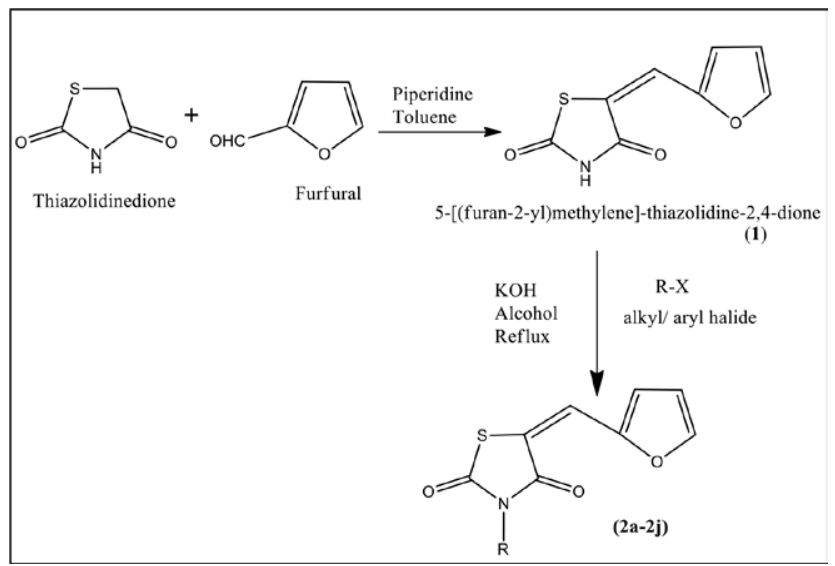

Figure 1: Scheme of synthesis of 3-substituted-5-[(furan-2-yl)methylene]-thiazolidine-2,4-dione derivatives. in $8 \mathrm{ml}$ of toluene. Reaction mixture was irradiated using RGSSIRR model Raga's microwave at 350W power level and $120^{\circ} \mathrm{C}$ temperature for about $8 \mathrm{~min}$. After the reaction completion, diluted with $1 \mathrm{M} \mathrm{HCl}$ and ice-water; obtained precipitate was filtered off, washed from cold water followed by dry toluene. Finally it was recrystallized from ethanol. ${ }^{27}$

5-[(furan-2-yl)-methylene]-thiazolidine-2,4-dione was obtained as yellow powder analyzed for the yield $68.58 \%$ (conventional synthesis), $82.51 \%$ (Microwave synthesis), $m p 230-232^{\circ} \mathrm{C}, \mathrm{R}_{\mathrm{f}}$ value is 0.52 (benzene and ethyl acetate $8: 2$ ratio). FTIR spectrum showed the characteristic intense bands in $\mathrm{cm}^{-1}$ at $3364.42(-\mathrm{NH}-)$, $1668.26(\mathrm{C}=\mathrm{O}), 1234.05(\mathrm{C}-\mathrm{N}), 2964.28(-\mathrm{CH}), 3029.61$ $(=\mathrm{C}-\mathrm{H}), 620.42(\mathrm{C}-\mathrm{S})$ and $1628.52(\mathrm{C}=\mathrm{C})$. The ${ }^{1} \mathrm{H}-\mathrm{NMR}$ spectrum showed the characteristic signals at $400 \mathrm{MHz}$

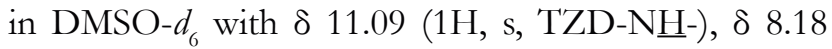
$(1 \mathrm{H}, \mathrm{s},=\mathrm{C} \underline{\mathrm{H}}-$ methylene $), 6.42-6.56(1 \mathrm{H}, \mathrm{t}$, furan-4'-H), 7.05-7.38 $(1 \mathrm{H}, \mathrm{d}$, furan-3'-H), 7.65-7.81 $(1 \mathrm{H}, \mathrm{d}$, furan5 '-H). The ${ }^{13} \mathrm{C}-\mathrm{NMR}$ spectrum showed the characteristic signals at $400 \mathrm{MHz}$ in DMSO- $d_{6}$ with $\delta 168.4, \delta 166.5$, $\delta 153.2, \delta 146.5, \delta 143.2, \delta 123.4, \delta$ 114.0, $\delta$ 111.5. The mass spectrum of the compound (2) was further confirms with ESI-MS: $\mathrm{m} / \mathrm{z}\left(\mathrm{M}^{+}\right) 195$.

\section{Synthesis of 3-substituted-5-[(furan-2-yl)-methylene]- thiazolidine-2,4-diones (2a-2j) \\ Conventional synthesis}

$0.01 \mathrm{~mol}$ ethanolic potassium hydroxide solution was added in drop wise to ethanolic suspension of $0.01 \mathrm{~mol}$ 5-[(furan-2-yl)-methylene]-thiazolidine-2,4-dione (1), stirred at for 15 to $20 \mathrm{~min}$ at room temperature. $0.01 \mathrm{~mol}$ aryl/ alkyl halide was added, refluxed for about 6-7.5 hr with stirring. Chromatographic TLC method was used to observe the reaction progress and completion (tolueneethylacetate; 7:3). Cool the reaction mixture, diluted with ice water. Further, obtained solid precipitate was filtered off, washed from cold water and diethyl ether. Crude product was recrystallized from ethanol. ${ }^{28}$

\section{Microwave synthesis}

A mixture of $2 \mathrm{mmol}$ anhydrous potassium carbonate in $5 \mathrm{ml}$ of acetone and $5 \mathrm{ml}$ of DMF and $0.01 \mathrm{~mol}$ 5-[(furan-2-yl)-methylene]-thiazolidine-2,4-dione (1) in acetone contents were stirred for about $20 \mathrm{~min}$ at room temperature. Add $0.01 \mathrm{~mol} \mathrm{Aryl} /$ alkyl halide and placed in RGSSIRR model Raga's microwave, irradiated at $300 \mathrm{~W}$ power level, $120^{\circ} \mathrm{C}$ temperature for about $10-12 \mathrm{~min}$. After the reaction completion, it was cooled, diluted with ice water, obtained solid precipitate was filtered off, washed from cold water and diethyl ether. Further it was purified by recrystallization from ethanol. ${ }^{29}$ 


\section{Spectral data of synthesized compounds (2a-2j)}

3-ethyl-5-[(furan-2-yl)-methylene]-thiazolidine-2,4dione (2a):- FTIR [ $\mathrm{cm}^{-1}$ ]: $3008.57(=\mathrm{C}-\mathrm{H}), 2936.45$ $(\mathrm{C}-\mathrm{H}), \quad 1750.15(\mathrm{C}=\mathrm{O}), \quad 1708.54 \quad(\mathrm{C}=\mathrm{O}), \quad 1228.50$ (C-N), 1658.24 (C=C), 1124.17 (C-O-C), 615.22 (C-S). ${ }^{1} \mathrm{H}-\mathrm{NMR}[8,400 \mathrm{MHz}]: 8.36(1 \mathrm{H}, \mathrm{s},=\mathrm{CH}-$ methylene), 6.72-6.98 (1H, t, furan-4'-H), 7.15-7.28 (1H, d, furan-3'-H), 7.90-8.01 (1H, d, furan-5'-H), 1.36-1.44 (3H, t, $\left.-\mathrm{CH}_{2}-\mathrm{CH}_{3}\right)$, 2.45-2.68 $\left(2 \mathrm{H}, \mathrm{m},-\mathrm{CH}_{2}-\mathrm{CH}_{3}\right) \cdot{ }^{13} \mathrm{C}-\mathrm{NMR}[\delta, 100 \mathrm{MHz}]$ : 175.1, 168.6, 153.3, 144.2, 141.1, 123.4, 114.1, 111.4, 36.5, 22.4. ESI-MS: $\mathrm{m} / \mathrm{z}\left[\mathrm{M}^{+}\right] 223$.

3-(2-aminoethyl)-5-[(furan-2-yl)-methylene]thiazolidine-2,4-dione (2b):- FTIR $\left[\mathrm{cm}^{-1}\right]: 3388.45$ (-NH-), 1768.24 (C=O), $1721.25 \quad(\mathrm{C}=\mathrm{O}), 1205.47$ (C-N), $2975.17(\mathrm{C}-\mathrm{H}), 3024.48$ (=C-H), $621.21(\mathrm{C}-\mathrm{S})$, 1640.47 (C=C), 1145.28 (C-O-C). ${ }^{1} \mathrm{H}-\mathrm{NMR}[\delta, 400$ $\mathrm{MHz}]: 8.41(1 \mathrm{H}, \mathrm{s},=\mathrm{C} \underline{\mathrm{H}}-$ methylene $), 6.66-6.85(1 \mathrm{H}$, $\mathrm{t}$, furan-4'-H), 7.03-7.41 (1H, d, furan-3'-H), 7.78-8.10 $\left(1 \mathrm{H}\right.$, d, furan-5'-H), 3.54-3.81 (2H, t, $\left.-\mathrm{CH}_{2}-\mathrm{CH}_{2}-\mathrm{NH}_{2}\right)$, 2.83-2.3.08 (2H, m, $\left.-\mathrm{CH}_{2}-\mathrm{CH}_{2}-\mathrm{NH}_{2}\right), 2.43-2.61(2 \mathrm{H}$, t, $\left.-\mathrm{CH}_{2}-\mathrm{CH}_{2}-\mathrm{NH}_{2}\right) \cdot{ }^{13} \mathrm{C}-\mathrm{NMR}[\delta, 100 \mathrm{MHz}]: 170.5,164.3$, $151.5,145.7,141.8,121.8,115.5,111.7,54.6,35.2$. ESI-MS: $\mathrm{m} / z\left[\mathrm{M}^{+}\right] 238$.

3-(2-aminoacetyl)-5-[(furan-2-yl)-methylene]thiazolidine-2,4-dione (2c):- FTIR [ $\left.\mathrm{cm}^{-1}\right]: 3315.57$ (-NH-), $1725.14(\mathrm{C}=\mathrm{O}), 1695.32(\mathrm{C}=\mathrm{O}), 1216.17(\mathrm{C}-\mathrm{N})$, 2958.22 (C-H), 3052.54 (=C-H), 619.52 (C-S), 1651.21 $(\mathrm{C}=\mathrm{C}), 1128.62$ (C-O-C). ${ }^{1} \mathrm{H}-\mathrm{NMR}[\delta, 400 \mathrm{MHz}]: 8.53$ $(1 \mathrm{H}, \mathrm{s},=\mathrm{C} \underline{\mathrm{H}}-\mathrm{methylene}), 6.52-6.75\left(1 \mathrm{H}, \mathrm{t}\right.$, furan- $\left.4^{\prime}-\mathrm{H}\right)$, 7.14-7.22 $(1 \mathrm{H}, \mathrm{d}$, furan-3'-H), 7.53-7.63 $(1 \mathrm{H}, \mathrm{d}$, furan5'-H), $4.14\left(2 \mathrm{H}, \mathrm{s},-\mathrm{CH}_{2}-\mathrm{CO}-\mathrm{NH}_{2}\right), 5.27\left(2 \mathrm{H}, \mathrm{s},-\mathrm{CH}_{2}-\right.$ CO-N $\left.\underline{\mathrm{H}}_{2}\right) \cdot{ }^{13} \mathrm{C}-\mathrm{NMR}[\delta, 100 \mathrm{MHz}]: 175.5,168.1,165.6$, 153.2, 146.8, 142.7, 123.9, 114.9, 110.3, 48.6. ESI-MS: $\mathrm{m} / \mathrm{z}\left[\mathrm{M}^{+}\right] 252$.

3-(4-bydroxyphenyl)-5-[(furan-2-yl)-methylene]thiazolidine-2,4-dione (2d):- FTIR $\left[\mathrm{cm}^{-1}\right]: 3555.14$ $(-\mathrm{OH}), 1716.05(\mathrm{C}=\mathrm{O}), 1684.42(\mathrm{C}=\mathrm{O}), 1228.61(\mathrm{C}-\mathrm{N})$, 2968.17 (C-H), 3034.11 (=C-H), 622.42 (C-S), 1669.75 $(\mathrm{C}=\mathrm{C}), 1137.82$ (C-O-C). ${ }^{1} \mathrm{H}-\mathrm{NMR}[\delta, 400 \mathrm{MHz}]: 8.46$ $(1 \mathrm{H}, \mathrm{s},=\mathrm{C} \underline{\mathrm{H}}-$ methylene $), 6.35-6.61(1 \mathrm{H}, \mathrm{t}$, furan-4'-H), 7.24-7.59 $(1 \mathrm{H}, \mathrm{d}$, furan-3'-H), 7.71-7.92 $(1 \mathrm{H}, \mathrm{d}$, furan5'-H), $5.74(1 \mathrm{H}, \mathrm{s},-\mathrm{OH}), 6.54-6.79$ (2H, d, phenyl 3'- $\mathrm{H}$ and 5'-H), 7.41-7.62 (2H, d, phenyl 2'-H and 6'-H). ${ }^{13} \mathrm{C}-$ NMR [8, $100 \mathrm{MHz}]$ : 178.4, 167.1, 156.8, 153.6, 148.2, 144.5, 128.4, 125.7, 123.8, 118.2, 114.1, 112.4. ESI-MS: $\mathrm{m} / \mathrm{z}\left[\mathrm{M}^{+}\right] 287$.

3-(4-aminophenyl)-5-[(furan-2-yl)-methylene]thiazolidine-2,4-dione (2e):-FTIR [ $\left.\mathrm{cm}^{-1}\right]: 3321.02(-\mathrm{NH}-$ ), $1730.17(\mathrm{C}=\mathrm{O}), 1699.21(\mathrm{C}=\mathrm{O}), 1247.57(\mathrm{C}-\mathrm{N}), 2974.33$ (C-H), 3082.11 (=C-H), 620.08 (C-S), 1648.69 (C=C), 1108.17 (C-O-C). ${ }^{1} \mathrm{H}-\mathrm{NMR}$ [8, $400 \mathrm{MHz}$ ]: 8.49 (1H, s,
$=\mathrm{C} \underline{\mathrm{H}}$ - methylene), $6.09-6.25$ (1H, t, furan-4'-H), 7.53-7.63 $(1 \mathrm{H}, \mathrm{d}$, furan-3'-H), 8.05-8.18 (1H, d, furan -5 '- $-\mathrm{H}), 4.68$ $\left(2 \mathrm{H}, \mathrm{s},-\mathrm{NH}_{2}\right), 6.41-6.53(2 \mathrm{H}, \mathrm{d}$, phenyl 3'- $\mathrm{H}$ and 5'-H), 7.32-7.45 (2H, d, phenyl 2'-H and $\left.6{ }^{\prime}-\mathrm{H}\right) .{ }^{13} \mathrm{C}-\mathrm{NMR}[\delta$, $100 \mathrm{MHz}]: 178.8,167.4,155.8,147.8,144.4,140.8,126.2$, 123.2, 120.4, 118.4, 115.7, 110.2. ESI-MS: $\mathrm{m} / z\left[\mathrm{M}^{+}\right] 286$. 3-(benzyl)-5-[(furan-2-yl)-methylene]-thiazolidine2,4-dione (2f):- FTIR [ $\left.\mathrm{cm}^{-1}\right]$ : $1729.64(\mathrm{C}=\mathrm{O}), 1695.09$ $(\mathrm{C}=\mathrm{O}), 1232.51(\mathrm{C}-\mathrm{N}), 2951.17(\mathrm{C}-\mathrm{H}), 3044.27(=\mathrm{C}-\mathrm{H})$, 618.10 (C-S), 1648.07 (C=C), 1122.59 (C-O-C). ${ }^{1} \mathrm{H}-\mathrm{NMR}$

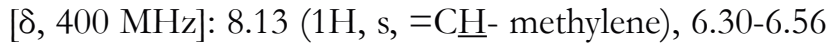
$(1 \mathrm{H}, \mathrm{t}$, furan-4'-H), 7.64-7.79 (1H, d, furan-3'-H), 7.85-7.99 $\left(1 \mathrm{H}, \mathrm{d}\right.$, furan-5'-H), $4.49\left(2 \mathrm{H}, \mathrm{s},-\mathrm{C}_{2}-\right.$ Phenyl), 7.10-7.48 $\left(5 \mathrm{H}, \mathrm{m}\right.$, phenyl). ${ }^{13} \mathrm{C}-\mathrm{NMR}[\delta, 100 \mathrm{MHz}]: 175.8,167.6$, 153.1, 149.2, 147.9, 144.6, 129.7, 126.4, 123.2, 121.6, 117.2, 113.2, 45.3. ESI-MS: $\mathrm{m} / z\left[\mathrm{M}^{+}\right] 285$.

3-(4-methoxyphenyl)-5-[(furan-2-yl)-methylene]thiazolidine-2,4-dione (2g):- FTIR [ $\left.\mathrm{cm}^{-1}\right]$ : 3064.67 (=C-H), $1739.32(\mathrm{C}=\mathrm{O}), 1697.35(\mathrm{C}=\mathrm{O}), 1234.17(\mathrm{C}-\mathrm{N})$, $2978.42(\mathrm{C}-\mathrm{H}), 619.64(\mathrm{C}-\mathrm{S}), 1658.23(\mathrm{C}=\mathrm{C}), 1151.97$ (C-O-C). ${ }^{1} \mathrm{H}-\mathrm{NMR}[\delta, 400 \mathrm{MHz}]: 8.56(1 \mathrm{H}, \mathrm{s},=\mathrm{C} \underline{\mathrm{H}}-$ methylene), 6.11-6.40 (1H, t, furan-4'-H), 7.37-7.48 $(1 \mathrm{H}$, d, furan-3'-H), 8.15-8.27 (1H, d, furan-5'-H), $3.72(3 \mathrm{H}, \mathrm{s}$, $\left.-\mathrm{OCH}_{3}\right), 6.53-6.67(2 \mathrm{H}$, d, phenyl 3'- $\mathrm{H}$ and 5'-H), 7.58$7.69\left(2 \mathrm{H}, \mathrm{d}\right.$, phenyl $2^{\prime}-\mathrm{H}$ and $\left.6^{\prime}-\mathrm{H}\right) .{ }^{13} \mathrm{C}-\mathrm{NMR}[\delta, 100$ $\mathrm{MHz}$ : 174.4, 166.2, 156.7, 151.5, 145.3, 141.5, 127.0, 124.4, 121.4, 117.7, 114.1, 112.4, 57.7. ESI-MS: $\mathrm{m} / \mathrm{z}\left[\mathrm{M}^{+}\right]$ 301.

3-[4-(dimethylamino)pbenyl]-5-[(furan-2-yl)methylene]-thiazolidine-2,4-dione (2b):- FTIR [ $\left.\mathrm{cm}^{-1}\right]$ : $1731.74(\mathrm{C}=\mathrm{O}), 1685.24(\mathrm{C}=\mathrm{O}), 1259.34(\mathrm{C}-\mathrm{N}), 2938.29$ (C-H), 3085.37 (=C-H), 628.36 (C-S), $1673.41(\mathrm{C}=\mathrm{C})$, 1138.46 (C-O-C). ${ }^{1} \mathrm{H}-\mathrm{NMR}$ [8, $\left.400 \mathrm{MHz}\right]: 8.19$ (1H, s, $=\mathrm{C} \underline{\mathrm{H}}$ - methylene), 6.84-6.93 (1H, t, furan-4'-H), 7.08-7.29 $\left(1 \mathrm{H}, \mathrm{d}\right.$, furan $\left.-3^{\prime}-\mathrm{H}\right), 7.79-7.88\left(1 \mathrm{H}, \mathrm{d}\right.$, furan $\left.-5^{\prime}-\mathrm{H}\right), 2.96$ (6H, s, -dimethylamino), 6.52-6.65 (2H, d, phenyl 3'- $-\mathrm{H}$ and 5 '- $-\mathrm{H}), 7.48-7.56\left(2 \mathrm{H}\right.$, d, phenyl 2 '- $-\mathrm{H}$ and $\left.6{ }^{\prime}-\mathrm{H}\right) .{ }^{13} \mathrm{C}-$

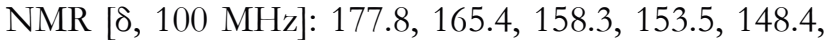
142.6, 128.6, 125.3, 122.7, 118.3, 115.8, 111.5, 42.5 . ESI-MS: $\mathrm{m} / z\left[\mathrm{M}^{+}\right] 314$.

3-(4-acetylpbenyl)-5-[(furan-2-yl)-methylene]thiazolidine-2,4-dione (2i):- FTIR [ $\left.\mathrm{cm}^{-1}\right]: 1715.62$ $(\mathrm{C}=\mathrm{O}), 1659.08(\mathrm{C}=\mathrm{O}), 1238.19(\mathrm{C}-\mathrm{N}), 2959.17(\mathrm{C}-\mathrm{H})$, 3066.26 (=C-H), 620.11 (C-S), 1685.22 (C=C), 1129.65 (C-O-C). ${ }^{1} \mathrm{H}-\mathrm{NMR}[\delta, 400 \mathrm{MHz}]: 8.34(1 \mathrm{H}, \mathrm{s},=\mathrm{C} \underline{\mathrm{H}}-$ methylene), 6.68-6.79 (1H, t, furan-4'-H), 7.11-7.32 $(1 \mathrm{H}$, d, furan-3'-H), 7.51-7.68 (1H, d, furan-5'-H), $2.58(3 \mathrm{H}, \mathrm{s}$, $\left.-\mathrm{COC} \underline{\mathrm{H}}_{3}\right), 7.84-7.96(2 \mathrm{H}$, d, phenyl 3'- $\mathrm{H}$ and 5'-H), 7.55$7.68\left(2 \mathrm{H}\right.$, d, phenyl 2'-H and 6'-H). ${ }^{13} \mathrm{C}-\mathrm{NMR}[\delta, 100$ $\mathrm{MHz}$ : 195.5, 175.2, 164.6, 153.8, 146.8, 142.3, 137.5, 132.1, 129.8, 125.5, 122.4, 114.6, 111.8, 30.8. ESI-MS: $\mathrm{m} / \mathrm{z}\left[\mathrm{M}^{+}\right] 313$. 
3-(p-tolyl)-5-[(furan-2-yl)-methylene]-thiazolidine2,4-dione (2j):- FTIR [ $\left.\mathrm{cm}^{-1}\right]: 3025.40(=\mathrm{C}-\mathrm{H}), 1737.19$ $(\mathrm{C}=\mathrm{O}), 1703.55(\mathrm{C}=\mathrm{O}), 1256.46(\mathrm{C}-\mathrm{N}), 2989.20(\mathrm{C}-\mathrm{H})$, 627.41 (C-S), 1674.57 (C=C), 1149.22 (C-O-C). ${ }^{1} \mathrm{H}-\mathrm{NMR}$

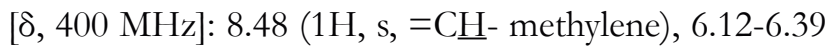
$(1 \mathrm{H}, \mathrm{t}$, furan-4'-H), 6.61-6.75 $(1 \mathrm{H}, \mathrm{d}$, furan-3'-H), 8.17-8.26 $\left(1 \mathrm{H}, \mathrm{d}\right.$, furan-5'-H), $2.52\left(3 \mathrm{H}, \mathrm{s},-\mathrm{CH}_{3}\right), 7.30-7.45(2 \mathrm{H}, \mathrm{d}$, phenyl 3'-H and 5'-H), 7.51-7.63 (2H, d, phenyl 2'-H and 6 '-H). ${ }^{13} \mathrm{C}-\mathrm{NMR}[\delta, 100 \mathrm{MHz}]: 177.2,168.7,152.4$, 146.3, 143.6, 135.1, 130.6, 128.2, 124.1, 122.8, 112.5, 110.2, 26.6. ESI-MS: $\mathrm{m} / \mathrm{z}\left[\mathrm{M}^{+}\right] 285$.

\section{In-vivo Hypoglycemic Activity Evaluation}

In-vivo hypoglycemic study was conducted on alloxan induced Wistar-Albino rats of either sex using tail tipping technique. ${ }^{30,31}$ Rats (170-200 g weight) were procured from Sainadh Animal Supplier Agencies, Hyderabad. Initially before commencement of experiments, rats were acclimatized in the animal house laboratory for 1 week, feed with pellet and water ad libitum. Rats were placed in cages for about $12 \mathrm{hr}$ dark cycle and $12 \mathrm{hrs}$ light cycle at room temperature. For about $24 \mathrm{hr}$, acclimatized animals were reserved for fasting with water ad libitum, intraperitoneally alloxan monohydrate $(120 \mathrm{mg} / \mathrm{kg})$ in normal saline solution was administered. After one hr of alloxan monohydrate administration animals were given ad libitum. For a day, in feeding bottle 5\% dextrose solution was given to control the premature hypoglycemic segment. Blood drop was collected from the tail vein portion, the blood glucose levels were measured with the help of digital Accu-Chek glucose monitor.

After $72 \mathrm{hr}$ of alloxination, the rats possess blood glucose levels beyond $150 \mathrm{mg} / \mathrm{dl}$ were grouped for the acute and chronic studies (each group consists of 6 rats, $n=6$ ). In the acute study, test sample equivalent to 200 $\mathrm{mg} / \mathrm{kg}$ as average human intake was considered $36 \mathrm{mg} /$ $\mathrm{kg}$ single dose. Test samples were given by oral route with $0.25 \%$ carboxy methyl cellulose solution. Standard rosiglitazone of $30 \mathrm{mg} / \mathrm{kg}$ body weight dose was given to the rats. A dose of $35 \mathrm{mg} / \mathrm{kg}$ body weight was given to the rats as test sample during acute study. At 0, 1, 2, 4, 6 and $8 \mathrm{hr}$ the blood samples were collected and measured the glucose levels of blood. By considering the acute study results, the test samples were particularly selected in the chronic study and doses of $35 \mathrm{mg} / \mathrm{kg}$ and $70 \mathrm{mg} /$ $\mathrm{kg}$ body weight was given to the rats. Blood glucose levels measured after 30 min of dose administration and considered $0^{\text {th }}$ day. Further the glucose levels of blood were measured on $7^{\text {th }}$ day and $15^{\text {th }}$ day.

During hypoglycaemic activity screening, study protocol approvals were taken from Institutional Animal Ethics Committee (IAEC)) and Committee for the Purpose of
Control and Supervision of Experiments on Animals (CPCSEA) with registration no.1847/PO/Re/S/16/ CPCSEA. Glucose levels of blood and body weights were mentioned as mean \pm standard error of mean (SEM). Values were analyzed from one-way analysis of variance (ANOVA) followed by Dunnet's $s$ t test.

\section{Molecular Docking Studies}

Numerous factors involved in selection of protein for docking studies for example structure must be determined through X-ray diffraction, 2.0 to $2.5 \AA$ resolution, consists of co-crystallized ligand, 3D structure of selected protein does not contain any protein breaks. ${ }^{32}$ Target receptor co-crystal structure was taken from protein data bank PDB ID-2PRG (2.3 Å resolution). Commonly used docking validation method was done by pose selection whereby docking programs used to re-dock into the target active site with a known conformation and orientation, typically from the co-crystal structure. Programs that were able to return poses below a preselected Root Mean Square Deviation (RMSD) value from the known conformation usually below 1.5 or $2 \AA$ depending on ligand size are considered to have performed successfully. Target protein was arranged by removing heteroatom portions, water molecules, nonreceptor atoms, other ions, etc. Thiazolidine-2,4-dione moiety containing anti-diabetic drugs possess activity through PPAR $\gamma$ receptor protein active site..$^{33}$ Designed compounds molecular docking studies was performed on selected target 2PRG for their potential PPAR $\gamma$ agonist property. ${ }^{34}$ Different ligands were modelled with the software ChemDraw Ultra 12.0 and transformed as appropriate $3 \mathrm{D}$ model structure, subjected to intend for energy minimization, which is necessary in the PDB files development and molecular docking. Docking was carried out on the designed ligands at the active site region of $2 \mathrm{PRG}$ using Auto-Dock 4.2.6. Binding interactions between diverse thiazolidine-2,4-dione ligands and 2PRG active site region was observed by considering active site as a rigid molecule and ligands being flexible. To predict the binding energy, docking was performed on prepared ligands at 2PRG active site region for best fit locations using rosiglitazone as standard reference ligand.

\section{RESULTS AND DISCUSSION}

\section{Chemistry}

Knoevenagel condensation of thiazolidine-2,4dione and furfural gives 5-[(furan-2-yl)-methylene]thiazolidine-2,4-dione (1). Further it was coupled with various alkyl/ aryl halides in alcoholic $\mathrm{KOH}$ to produce 
Table 1: Physical characterization data of synthesized compounds 2a-2j.

\begin{tabular}{|c|c|c|c|c|c|c|c|}
\hline \multirow{2}{*}{ Compound } & & m.p. $\left({ }^{\circ} \mathbf{C}\right)$ & \multirow{2}{*}{$\begin{array}{c}\text { Molecular } \\
\text { formula }\end{array}$} & $\begin{array}{c}\text { Molecular } \\
\text { weight }\end{array}$ & \multicolumn{2}{c|}{ Conventional synthesis } & \multicolumn{2}{|c|}{ Microwave synthesis } \\
\cline { 5 - 8 } & & & \% yield & reaction time & \% yield & reaction time \\
\hline $\mathbf{2 a}$ & $160-162$ & $\mathrm{C}_{10} \mathrm{H}_{9} \mathrm{NO}_{3} \mathrm{~S}$ & 223.25 & 68.48 & $6 \mathrm{hr}$ & 73.85 & $10 \mathrm{~min}$ \\
\hline $\mathbf{2 b}$ & $192-194$ & $\mathrm{C}_{10} \mathrm{H}_{10} \mathrm{~N}_{2} \mathrm{O}_{3} \mathrm{~S}$ & 238.26 & 66.63 & $6.5 \mathrm{hr}$ & 74.42 & $12 \mathrm{~min}$ \\
\hline $\mathbf{2 c}$ & $188-190$ & $\mathrm{C}_{10} \mathrm{H}_{8} \mathrm{~N}_{2} \mathrm{O}_{4} \mathrm{~S}$ & 252.25 & 65.59 & $7 \mathrm{hr}$ & 72.56 & $10 \mathrm{~min}$ \\
\hline $\mathbf{2 d}$ & $204-206$ & $\mathrm{C}_{14} \mathrm{H}_{9} \mathrm{NO}_{4} \mathrm{~S}$ & 287.29 & 72.45 & $7.5 \mathrm{hr}$ & 80.15 & $11 \mathrm{~min}$ \\
\hline $\mathbf{2 e}$ & $176-178$ & $\mathrm{C}_{14} \mathrm{H}_{10} \mathrm{~N}_{2} \mathrm{O}_{3} \mathrm{~S}$ & 286.31 & 65.30 & $6.5 \mathrm{hr}$ & 73.25 & $12 \mathrm{~min}$ \\
\hline $\mathbf{2 f}$ & $186-188$ & $\mathrm{C}_{15} \mathrm{H}_{11} \mathrm{NO}_{3} \mathrm{~S}$ & 285.32 & 67.28 & $7 \mathrm{hr}$ & 71.63 & $10 \mathrm{~min}$ \\
\hline $\mathbf{2 g}$ & $210-212$ & $\mathrm{C}_{15} \mathrm{H}_{11} \mathrm{NO}_{4} \mathrm{~S}$ & 301.32 & 71.61 & $7.5 \mathrm{hr}$ & 80.55 & $10 \mathrm{~min}$ \\
\hline $\mathbf{2 h}$ & $224-226$ & $\mathrm{C}_{16} \mathrm{H}_{14} \mathrm{~N}_{2} \mathrm{O}_{5} \mathrm{~S}$ & 314.36 & 70.74 & $7 \mathrm{hr}$ & 78.42 & $12 \mathrm{~min}$ \\
\hline $\mathbf{2 i}$ & $200 .-202$ & $\mathrm{C}_{16} \mathrm{H}_{11} \mathrm{NO}_{4} \mathrm{~S}$ & 313.33 & 68.48 & $6.5 \mathrm{hr}$ & 77.28 & $11 \mathrm{~min}$ \\
\hline $\mathbf{2 j}$ & $194-196$ & $\mathrm{C}_{15} \mathrm{H}_{11} \mathrm{NO}_{3} \mathrm{~S}$ & 285.32 & 71.26 & $7 \mathrm{hr}$ & 78.45 & $12 \mathrm{~min}$ \\
\hline
\end{tabular}

\begin{tabular}{|c|c|c|c|c|c|c|}
\hline \multirow{2}{*}{ Compound } & \multicolumn{6}{|c|}{ Mean \pm SEM of Blood glucose level $\mathrm{mg} / \mathrm{dl}$} \\
\hline & $0 \mathrm{hr}$ & $1 \mathrm{hr}$ & $2 \mathrm{hr}$ & $4 \mathrm{hr}$ & $6 \mathrm{hr}$ & $8 \mathrm{hr}$ \\
\hline Normal & $123.22 \pm 2.18$ & $121.58 \pm 3.04$ & $121.47 \pm 2.45$ & $122.23 \pm 3.21$ & $121.52 \pm 4.07$ & $122.54 \pm 2.63$ \\
\hline Standard & $396.55 \pm 4.12^{*}$ & $250.44 \pm 4.11^{* *}$ & $193.33 \pm 3.52$ & $155.84 \pm 3.42^{* *}$ & $110.36 \pm 3.18$ & $103.32 \pm 3.51^{*}$ \\
\hline $2 a$ & $330.5 \pm 3.28^{*}$ & $294.11 \pm 4.56^{*}$ & $238.61 \pm 5.16^{*}$ & $212.31 \pm 4.35$ & $238.12 \pm 5.42$ & $265.34 \pm 3.56^{*}$ \\
\hline $2 b$ & $329.18 \pm 5.11^{*}$ & $301.3 \pm 4.45$ & $288.15 \pm 3.12$ & $270.41 \pm 2.62$ & $260.61 \pm 3.32^{* *}$ & $285.14 \pm 2.65$ \\
\hline 2c & $335.6 \pm 3.48^{*}$ & $310.51 \pm 2.21$ & $295.4 \pm 2.68^{*}$ & $276.42 \pm 4.64^{* *}$ & $258.4 \pm 2.08$ & $275.5 \pm 5.35^{*}$ \\
\hline 2d & $320.55 \pm 4.22$ & $283.2 \pm 5.48^{* *}$ & $210.61 \pm 5.32^{*}$ & $159.51 \pm 6.35$ & $118.54 \pm 4.31^{* *}$ & $106.61 \pm 2.46$ \\
\hline $2 e$ & $349.46 \pm 3.64$ & $315.3 \pm 2.67$ & $265.28 \pm 4.65^{\star *}$ & $241.65 \pm 2.84$ & $256.94 \pm 2.61^{*}$ & $279.15 \pm 2.69^{* *}$ \\
\hline $2 f$ & $338.66 \pm 3.54^{* *}$ & $304.64 \pm 5.09^{*}$ & $279.35 \pm 4.62^{* *}$ & $260.19 \pm 2.64$ & $275.27 \pm 4.35^{*}$ & $290.53 \pm 3.47^{*}$ \\
\hline $2 g$ & $325.24 \pm 4.27^{*}$ & $273.64 \pm 3.51^{*}$ & $199.46 \pm 3.62$ & $150.33 \pm 4.49^{*}$ & $120.46 \pm 4.38^{* *}$ & $105.63 \pm 3.38$ \\
\hline $2 \mathrm{~h}$ & $332.18 \pm 3.68^{*}$ & $292.3 \pm 2.34$ ** & $279.64 \pm 3.09$ & $255.46 \pm 4.81^{*}$ & $263.31 \pm 5.34$ & $282.06 \pm 4.35$ \\
\hline $2 i$ & $320.0 \pm 3.91^{* *}$ & $301.26 \pm 3.38^{*}$ & $295.42 \pm 5.06$ & $268.38 \pm 3.18^{*}$ & $275.25 \pm 2.67^{* *}$ & $300.57 \pm 4.11^{*}$ \\
\hline $2 j$ & $328.16 \pm 2.34$ & $297.42 \pm 4.67^{*}$ & $278.3 \pm 2.62$ & $255.38 \pm 3.38^{* *}$ & $264.39 \pm 3.65$ & $285.26 \pm 2.15$ \\
\hline
\end{tabular}

Standard Drug: Rosiglitazone; Statistical analysis was done by One-way ANOVA followed by Dunnet's ' $t$ ' test.

** $P<0.01$ (considered as significant), $* P<0.001$.

various derivatives $2 \mathrm{a}-2 \mathrm{j}$ (Figure 1). The titled compounds were developed by conventional and microwave assisted synthesis. Comparative study and physical characterization results of conventional and microwave synthesis were given in Table 1.

\section{In-vivo hypoglycaemic efficacy}

According to the above mentioned animal study protocols, acute study results were mentioned in Table 2 with respect to standard rosiglitazone stated that significant activity was shown by compounds $2 \mathrm{~d}$ and 2 g. Results of chronic study were mentioned in Table 3 stated that significant activity was shown at a dose of 70 $\mathrm{mg} / \mathrm{kg}$ body weight by the compounds $2 \mathrm{~d}$ and $2 \mathrm{~g}$.

\section{Molecular docking results}

Docking was done to rationalize the experimental biological activity. Docking was performed by means of recently updated version Auto-Dock dock engine 4.2.6 software. Co-crystallized ligand rosiglitazone was used for docking validation. It was extracted from $2 \mathrm{PRG}$ and redocked the co-crystallized ligand into the pose of active site receptor protein. Validation was performed by computing the root mean square deviation (RMSD) value by overlying the structures of co-crystallized ligand and redocked ligand. The RMSD value was found to be $1.28 \AA$, suggested that method was valid enough to be used for docking studies of other compounds. Binding interactions were observed in Pymol 1.7.4.5. Binding energy (in $\mathrm{kcal} / \mathrm{mol}$ ), hydrogen bonds, hydrogen bond lengths, number of hydrogen bonds and interacted amino acid residues were recognized at the target protein active site, the values were given in Table 4. Standard ligand rosiglitazone showed $-8.32 \mathrm{kcal} / \mathrm{mol}$ binding energy; forms five hydrogen bonds with bond lengths $3.19,3.28,2.54,2.38,4.11$ and identified the interacted 
Table 3: Effect of compounds $2 \mathrm{~d}$ and $2 \mathrm{~g}$ on fasting blood glucose level and body weight of alloxan induced diabetic rats (Chronic Study 15 days).

\begin{tabular}{|c|c|c|c|c|c|c|}
\hline \multirow{2}{*}{ Compound } & \multicolumn{3}{|c|}{ Blood glucose in mg/dl } & \multicolumn{3}{|c|}{ Body Weight in g } \\
\hline & Day 0 & Day 7 & Day 15 & Day 0 & Day 7 & Day 15 \\
\hline Standard & $358.42 \pm 4.13$ & $215.25 \pm 3.62^{\star *}$ & $164.34 \pm 2.11^{*}$ & $196.5 \pm 3.67$ & $194.68 \pm 2.08^{*}$ & $195.16 \pm 3.68^{*}$ \\
\hline $2 \mathrm{~d}(35 \mathrm{mg} / \mathrm{kg} \mathrm{bw})$ & $368.09 \pm 2.64^{*}$ & $230.27 \pm 3.31^{*}$ & $182.14 \pm 3.65^{\star}$ & $195.94 \pm 2.68^{*}$ & $196.68 \pm 4.61^{* *}$ & $195.35 \pm 2.95^{*}$ \\
\hline $2 \mathrm{~d}(70 \mathrm{mg} / \mathrm{kg} \mathrm{bw})$ & $354.32 \pm 4.68$ & $210.52 \pm 2.09^{*}$ & $167.34 \pm 3.18$ & $196.38 \pm 2.31^{*}$ & $195.38 \pm 2.41^{*}$ & $196.68 \pm 4.68$ \\
\hline $2 \mathrm{~g}(35 \mathrm{mg} / \mathrm{kg} \mathrm{bw})$ & $350.44 \pm 3.36^{*}$ & $230.49 \pm 3.27$ & $188.62 \pm 4.68^{*}$ & $197.61 \pm 2.07$ & $195.35 \pm 3.89^{* *}$ & $195.46 \pm 2.95^{*}$ \\
\hline $2 \mathrm{~g}(70 \mathrm{mg} / \mathrm{kg} \mathrm{bw})$ & $349.38 \pm 3.21^{*}$ & $225.49 \pm 2.64^{*}$ & $165.24 \pm 2.85^{*}$ & $196.42 \pm 2.63^{*}$ & $196.38 \pm 3.69^{*}$ & $194.2 \pm 1.65^{*}$ \\
\hline
\end{tabular}

Standard Drug: Rosiglitazone; Statistical analysis is done by One-way ANOVA followed by Dunnet's ' $t$ ' test.

** $P<0.01$ (considered as significant), $* P<0.001$.

\begin{tabular}{l}
\multicolumn{6}{|c|}{ Table 4: Binding energy and amino acid residues interacted by the compounds 2a-2j with the target PPAR $\gamma$} \\
protein PDB ID - 2PRG.
\end{tabular}

amino acids Gln286, Tyr473, His323, Arg288, His449, Met364, Cys285, Ser289. Target PDB ID- 2PRG protein 3D-structure was given in Figure 2.

The interaction complex of standard ligand rosiglitazone at with $2 \mathrm{PRG}$ active site was mentioned in Figure 3. Compound $2 \mathrm{~d}$ produced binding energy $-9.02 \mathrm{kcal} / \mathrm{mol}$; forms nine hydrogen bonds with bond lengths 3.32, $2.39,2.80,3.45,3.30,3.61,2.87,2.87,3.87$ and identified the interacted amino acids Tyr473, Ser289, His323, Tyr327, His449, Leu330, Cys285 were represented in Figure 4. Compound $2 \mathrm{~g}$ produced binding energy $-8.61 \mathrm{kcal} / \mathrm{mol}$; forms four hydrogen bonds with bond lengths $2.88,1.74,3.18,4.26$ and identified the interacted amino acids His449, Arg288, Val339, Phe282 were represented in Figure 5. Designed ligands $2 \mathrm{~d}$ and $2 \mathrm{~g}$

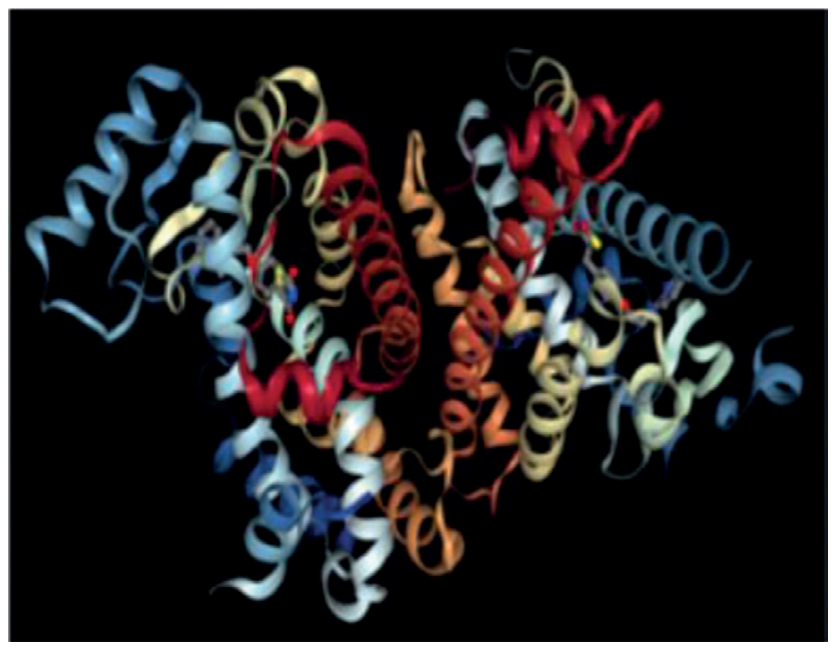

Figure 2: PPAR $_{\mathrm{Y}}$ protein 3D-structure from PDB ID- 2PRG. 


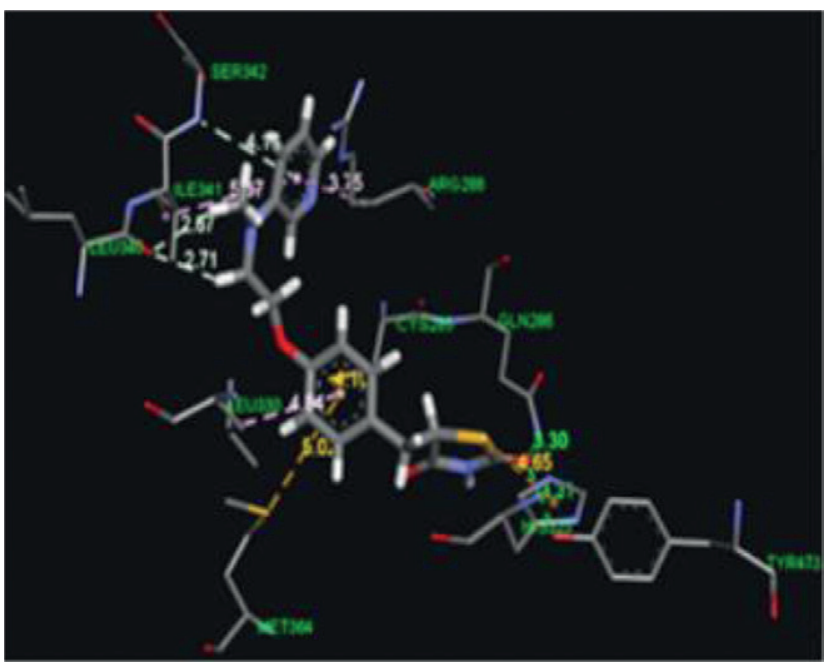

Figure 3: Binding mode of Rosiglitazone at $\mathrm{PPAR}_{\mathrm{y}}$ protein PDB ID-2PRG active site.

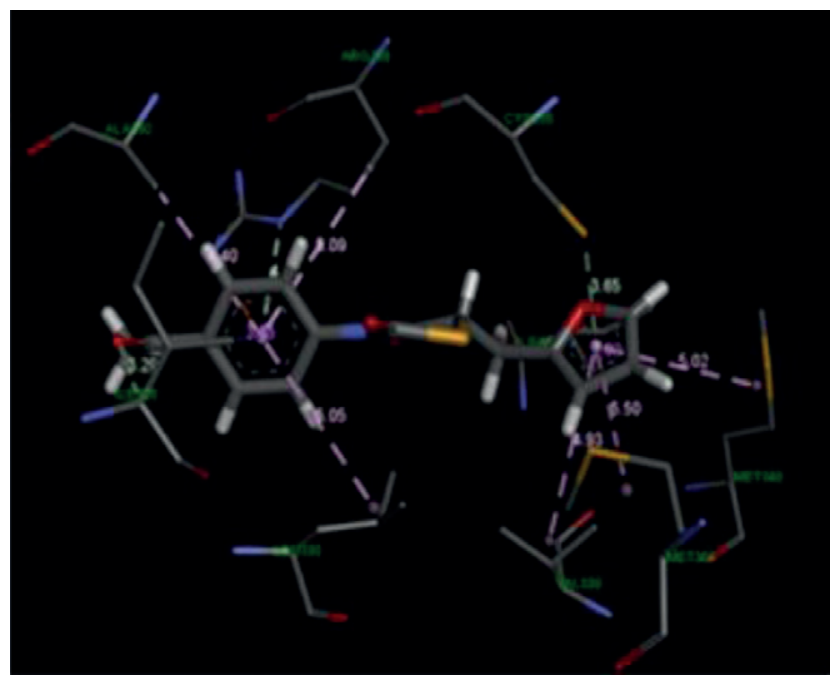

Figure 4: Binding mode of compound 2d at PPAR $_{y}$ protein PDB ID- 2PRG active site.

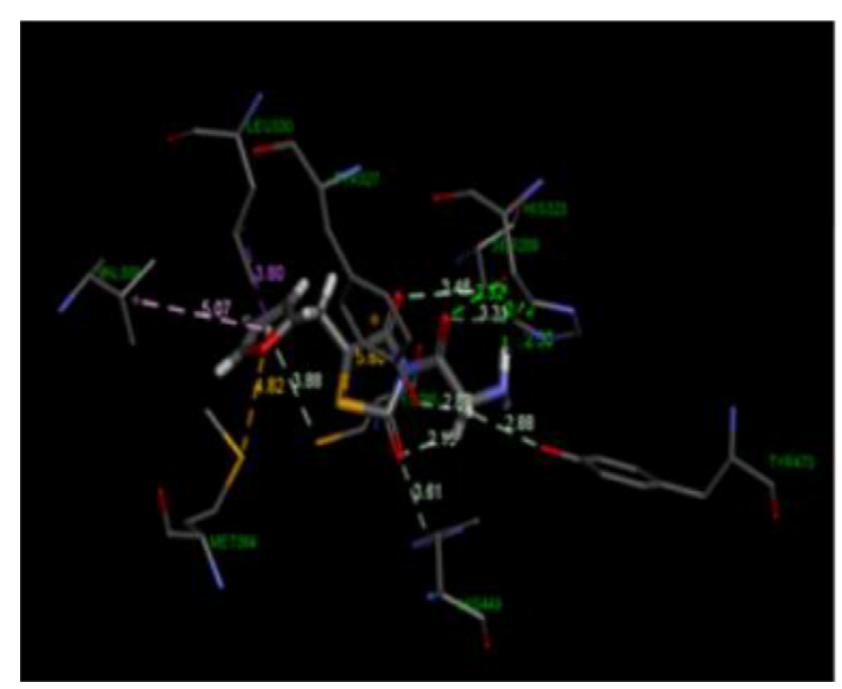

Figure 5: Binding mode of compound $2 \mathrm{~g}$ at PPAR id-2PRG active site. shown promising binding affinity with binding energy -9.02 and $-8.61 \mathrm{kcal} / \mathrm{mol}$ respectively in comparison to the standard rosiglitazone.

\section{CONCLUSION}

In this investigation an efficient versatile approach have been described to obtain various analogues of 3-substituted-5-[(furan-2-yl)-methylene]-thiazolidine2,4-diones possessing alkyl/ aryl substitution at $3^{\text {rd }}$ position and furyl methylene substitution at $5^{\text {th }}$ position was developed. Titled compounds $2 \mathrm{a}-2 \mathrm{j}$ were designed, synthesized with electron releasing and electron withdrawing groups by conventional and microwave synthesis methods. Microwave irradiation synthesis produced high yields at less reaction time intervals than in conventional synthesis. Spectrally and physically the compounds were characterized. The rationale of experimental in-vivo hypoglycemic activity revealed that electron releasing groups $\left(-\mathrm{OH}\right.$ and $\left.-\mathrm{OCH}_{3}\right)$ containing compounds $2 \mathrm{~d}$ and $2 \mathrm{~g}$ found to possess significant in-vivo hypoglycemic activity in both acute study, chronic study. Even the molecular docking studies revealed that, electron releasing groups containing compounds $2 \mathrm{~d}$ and $2 \mathrm{~g}$ displayed significant binding affinity at the 2 PRG PPAR $\gamma$ receptor protein active site. In $2 \mathrm{~d}$ and $2 \mathrm{~g}$ compounds, at $3^{\text {rd }}$ position presence of $-\mathrm{OH}$ and $-\mathrm{OCH}_{3}$ electron donating groups on the phenyl moiety may possibly considered as beneficial potential compounds.

\section{ACKNOWLEDGEMENT}

The authors declare that there is no funding source to carry out the research work. All the authors express gratitude towards the management of V. V. Institute of Pharmaceutical Sciences, Gudlavalleru, Andhra Pradesh, India for providing necessary facilities to carry out this work.

\section{CONFLICT OF INTEREST}

Authors declared no conflicts of interest.

\section{ABBREVIATIONS}

PPAR $\gamma:$ Peroxisomal proliferator activated receptors gamma type; PDB: Protein data bank; DM: Diabetes mellitus, TZD: Thiazolidine-2,4-dione; TLC: Thin layer chromatography; ${ }^{1} \mathbf{H}-\mathbf{N M R}$ : Proton Nuclear magnetic resonance; ${ }^{13} \mathbf{C}-\mathbf{N M R}$ : C-13 Nuclear magnetic resonance; DMF: Dimethyl formamide; DMSO: Dimethyl sulfoxide; IR: Infra-red; m.p.: Melting point; IAEC: Institutional Animal Ethics Committee; CPCSEA: Committee for 
the Purpose of Control and Supervision of Experiments on Animals; ANOVA: Analysis of variance; RMSD: Root Mean Square Deviation; SEM: Mean \pm standard error of mean.

\section{REFERENCES}

1. Hameed I, Shariq RM, Shahnaz AM, Mudasar N, Khalid G, Bashir AG. Type-2 diabetes mellitus: From a metabolic disorder to an inflammatory condition. World Journal of Diabetes. 2015;6(4):598-612.

2. American Diabetes Association. Diagnosis and classification of diabetes mellitus. Diabetes Care. 2008;31(1):S62-7.

3. Patil R, Gothankar J. Risk factors for type-2 diabetes mellitus: An urban perspective. Indian Journal of Medical Sciences. 2019;71(1):16-21.

4. Viberti G. Thiazolidinediones-benefits on microvascular complications of type-2 diabetes. Journal of Diabetes Complications. 2005;19(3):168-77.

5. Josephine FM, Cooper ME. Mechanisms of diabetic complications. Physiological Reviews. 2013;93(1):137-88.

6. Lee $\mathrm{CH}$, Olson P, Evans RM. Minireview: Lipid metabolism, metabolic diseases and peroxisome proliferator-activated receptors. Endocrinology. 2003;144(6):2201-7.

7. Davidson MA, Donald RM, Laurent AK. Thiazolidinedione drugs in the treatment of type 2 diabetes mellitus: Past, present and future. Journal of Critical Reviews in Toxicology. 2018;48(1):52-108.

8. Bansal G, Singh S, Monga V, Thanikachalam PV, Chawla P. Synthesis and biological evaluation of thiazolidine-2, 4-dione-pyrazole conjugates as antidiabetic, anti-inflammatory and antioxidant agents. Bioorganic Chemistry. 2019;92:103271.

9. Sabina $\mathrm{Y}$, Fabio C, Antonio L, Fabrizio DP, Fulvio L, Viswanathan V, et al. Novel benzylidene thiazolidinedione derivatives as partial PPARY agonists and their antidiabetic efects on Type 2 diabetes Scientific Reports. 2017;7:14453.

10. Pranav KP, Sivakumar PM. Protein tyrosine phosphatase-1B inhibitors: A novel therapeutic strategy for the management of type- 2 diabetes mellitus. Current Pharmaceutical Design. 2019;25(23):2526-39.

11. Sant KV, Yatesh SY, Suresh T. 2,4-Thiazolidinediones as PTP-1B Inhibitors: A Mini Review (2012-2018). Mini-Reviews in Medicinal Chemistry. 2019;19(7):591-8.

12. Insun $\mathrm{Y}$, Dubok $\mathrm{C}$, Lee HK, Cho H. Synthesis and biological evaluation of new benzylidenethiazolidine-2,4-dione derivatives as 15-hydroxyprostaglandin dehydrogenase inhibitors to control the intracellular levels of prostaglandin- $E_{2}$ for wound healing. Biotechnology and Bioprocess Engineering. 2019;24(3):464-75.

13. Abbas A, Mohsen K, Simin S, Elnaz S, Babak NN. Synthesis and evaluation of the hypoglycemic and hypolipidemic activity of novel arylidene thiazolidinedione analogs on a type 2 diabetes model. Pharmaceutical Chemistry Journal. 2016;50(3):165-71.

14. Mohammed IAK, Ashraf YK, Mallikarjun BK, Ningaraddi SB, Young-Dae G, Imitiyaz Ahmed MK. Synthesis, hypoglycemic and hypolipidemic activities of novel thiazolidinedione derivatives containing thiazole/triazole/oxadiazole ring. European Journal of Medicinal Chemistry. 2012;53:308-15.

15. Jyoti P, Arshad A, Arun KG. Synthesis and evaluation of some new 2-(5-(4-benzamidobenzylidene)-2,4- dioxothiazolidin-3-yl)acetic acid analogs as aldose reductase inhibitors. Asian Journal of Pharmaceutical and Clinical Research. 2017;10(1):62-6.

16. Kamel M, Harris P, Dimitris K, Luca Q, Vito C, Concettina LM, et al. Novel quinazolinone-based 2,4-thiazolidinedione-3-acetic acid derivatives as potent aldose reductase inhibitors. Future Medicinal Chemistry. 2017;9(18):2147-66.
17. Rena MP, Marc YD, Derek LR, Gil L. Anti-inflammatory agents in the treatment of diabetes and its vascular complications. Diabetes Care. 2016;39(2):S244-52.

18. Eleni X, Eleni-Margarita T, Athanasia P, Andreas M. Anti-inflammatory properties of antidiabetic agents. World Journal of Meta-Analysis. 2019;7(4):129-41.

19. Cristina MN, Mihaela D, Adrian P, Laurian V, Brindusa T, Ovidiu O. Development of new 5-(chromene-3-yl) methylene-2,4-thiazolidinediones as antimicrobial agents. Clujul Medical. 2016;89(1):122-7.

20. Nazar T, Agata P, Justyna Z, Grazyna G, Agata P, Monika W. Synthesis and in vitro antiproliferative and antibacterial activity of new thiazolidine-2,4dione derivatives. Journal of Enzyme Inhibition and Medicinal Chemistry. 2018;33(1):17-24.

21. Mohd JN, Jahangir AM, Shujauddin A, Farah N, Neelima S, Meeta S, et al. Therapeutic journey of 2,4-thiazolidinediones as a versatile scaffold: An insight into structure activity relationship. European Journal of Medicinal Chemistry. 2017;129:218-50.

22. Sucheta TS, Verma PK. Biological potential of thiazolidinedione derivatives of synthetic origin. Chemistry Central Journal. 2017;11(1):130.

23. Rekha S, Chandrashekhara S. Antioxidant and antibacterial activities of thiazolidinedione derivatives. International Journal of Pharmaceutical Sciences and Research. 2015;6(1):421-8.

24. Mishra G, Narsingh S, Pooja C. Synthesis and evaluation of thiazolidinedionecoumarin adducts as antidiabetic, anti-inflammatory and antioxidant agents. Letters in Organic Chemistry. 2015;12(6):429-45.

25. Cigdem O, Meltem CU, Nazanin A, Mehmet O, Oya BD. Thiazolidinedione or rhodanine: A study on synthesis and anticancer activity comparison of novel thiazole derivatives. Journal of Pharmacy and Pharmaceutical Sciences. 2017;20(1):415-27.

26. Gilles $B$, Abed $A A A Q$, Claes $D E$, et al. Synthesis and evaluation of thiazolidinedione and dioxazaborocane analogues as inhibitors of Al-2 quorum sensing in Vibrio harveyi. Bioorganic and Medicinal Chemistry. 2013;21(3):660-7.

27. Gabriel M, loana I, Adrian $\mathrm{P}$, et al. Microwave assisted synthesis of 3,5-disubstituted thiazolidine-2,4-diones with antifungal activity. Design, Synthesis, Virtual and in vitro Antifungal Screening. Farmica. 2017;65(3):414-22.

28. Swathi N, Ananda KTD, Subrahmanyam CVS, Satyanarayana K. Synthesis and in silico drug likeness evaluation of N,5-disubstituted-1,3-thiazolidine-2,4dione analogues. Journal of Pharmacy Research. 2013;6(1):107-11.

29. Marc G, Stana A, Adrian Pirnau A, et al. 3,5-Disubstituted thiazolidine-2,4diones: design, microwave-assisted synthesis, antifungal activity and ADMET screening. SLAS Discovery. 2018;23(8):807-14.

30. Anna PGN, Dipali D, Hemant DU. Facile synthesis and in vivo hypoglycemic activity of novel 2,4-hiazolidinedione derivatives. European Journal of Experimental Biology. 2012;2(2):343-53.

31. Shashikant RP, Prajact K, Ashwini P, Ana N, Kittur BS. Studies on the Synthesis of novel 2,4-thiazolidinedione derivatives with antidiabetic activity. Iranian Journal of Pharmaceutical Sciences. 2009;5(4):225-30.

32. Madhuri M, Prasad $\mathrm{CH}$, Vasudeva RA. In silico protein-ligand docking studies on thiazolidinediones as potential anticancer agents. International Journal of Computer Applications. 2014;95(6):13-6.

33. Arifa B, Shaheen B, Prasad KVSRG, Bharathi K. In-silico studies on functionalized azaglycine derivatives containing original article 2,4-thiazolidinedione scaffold on multiple targets. International Journal of Pharmacy and Pharmaceutical Sciences. 2017;9(8):209-15.

34. Akhiles R, Mahesh RD, Shilpa SH, et al. Synthesis, molecular docking studies and biological evaluation of 5-[4-(substituted)benzylidene or benzyl]thiazolodine-2,4-dione with their oral hypoglycaemic activity. International Research Journal of Pharmacy. 2013;4(8):151-7. 
PICTORIAL ABSTRACT

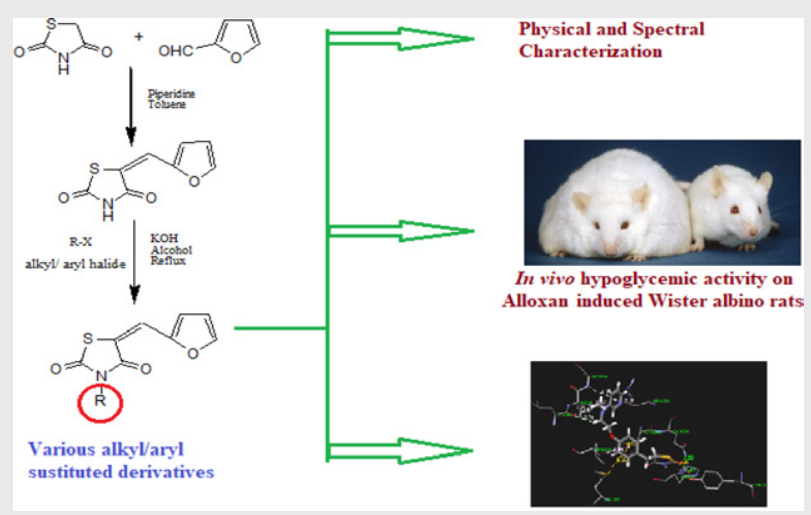

\section{SUMMARY}

Thiazolidine-2,4-dione scaffold is a promising pharmacophore as PPAR $\gamma$ receptor agonist. In view of this, designed and synthesized various thiazolidine-2,4-diones possessing alkyl/ aryl substitution at $3^{\text {rd }}$ position and furyl methylene substitution at $5^{\text {th }}$ position.

Microwave irradiation synthesis produced high yield at less reaction time in comparison to conventional synthesis.

In-vivo hypoglycemic activity screening, molecular docking studies revealed only two compounds shown promising hypoglycemic activity and good binding affinity at PPAR $\gamma$ receptor protein PDB ID- 2PRG.

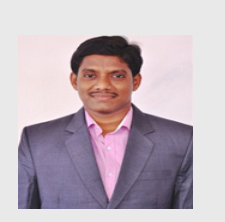

About Authors

Dr. Karumanchi Srikanth Kumar working as Associate Professor, Head, Department of Pharmaceutical Chemistry, V. V. Institute of Pharmaceutical Sciences, Gudlavalleru, Krishna District, AP, India. He has done his B.Pharmacy from Chalapathi Institute of Pharmaceutical Sciences, Guntur, AP. He did M.Pharmacy from Shri Vishnu College of Pharmacy, Bhimavaram, AP. He obtained his Ph.D degree in Pharmaceutical Sciences from Krishna University, Machilipatnam, AP. He has presented Scientific papers in various National \& International Seminars. He is author of 14 research articles in National and International Peer Reviewed Journals. His area of research interest towards synthesis, characterization, biological evaluation and molecular docking studies of some novel heterocyclic compounds.

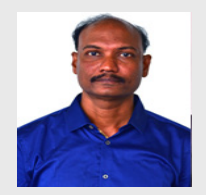

Dr. A. Lakshmana Rao is working as a Professor and Principal of V. V. Institute of Pharmaceutical Sciences, Gudlavalleru, A.P., India. He is having 20 years of Teaching, Research and Administrative experience. He guided 9 Ph.D. Scholars and 50 M.Pharm. Candidates. He received 30 Awards and Prizes from various International and National organizations. He received Eminent Pharmacist Award, Certificate of Excellence Award, Dr.APJ Abdul Kalam Award for Teaching Excellence, Chennai, Noble Principal of the Year Award and Principal of the Year Award from various International and National Organizations. He conferred with Meritorious University Best Teacher Award from Jawaharlal Nehru Technological University Kakinada, Kakinada. He is given 10 Guest Lectures in different universities and colleges. He published 319 papers in various reputed International and National Journals. He published 1 Text Book and 2 E-Books. He is Editor-in-Chief, Reviewer and Editorial Board Member for 114 reputed International and National journals. He organized 39 Seminars, Conferences and Workshops. He participated 92 Seminars, Conferences and Workshops. He presented 33 scientific papers in various Seminars and Conferences and received 12 prizes. He is having 1 Indian Patent in his credit. He received Grants from AICTE and $\mathrm{PCl}$. He is having memberships in various professional societies like IPA, APTI, OPF, ABAP, APP, ISTE etc.

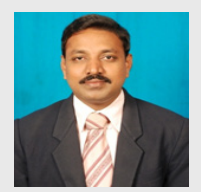

Dr. D. Rama Sekhara Reddy working as Head, Department of Chemistry, Controller of Examinations (I/C), Krishna University, Machilipatnam, Krishna District, AP, India. He has enormous administrative experience at various levels of the University. He has done M.Sc. Organic Chemistry from Acharya Nagarjuna University, Guntur, AP, India. He did Ph.D. in Organic Chemistry from Andhra University, Visakhapatnam, AP, India. He is a Post Doctoral Fellow from 2009-2011 at Institute of Biological Chemistry, Washington State University, Pullman, WA, USA. He is the Post Doctoral Research Associate from 2011-2012 at Centre for Pharmacology, Pharmaceuticals and Pharmacology, I-AIM, Bangalore, KA, India. He has presented Scientific papers in various National \& International Seminars. He is the author of 30 research articles in National and International Peer Reviewed Journals. His area of research interest includes Medicinal chemistry, Terrestrial natural products, Synthetic Organic Chemistry and Synthetic Methodologies.

Cite this article: Kumar KS, Rao AL, Reddy DRS. Design, Synthesis, Hypoglycemic Activity and Molecular Docking Studies of 3-substituted-5-[(furan-2-yl)-methylene]-thiazolidine-2,4-dione Derivatives. Indian J of Pharmaceutical Education and Research. 2021;55(1):266-75. 